\section{PTU-089 IS HELICOBACTER PYLORI ANTIBIOTIC RESISTANCE SURVEILLANCE NEEDED AND HOW CAN IT BE DELIVERED? A FEASIBILITY STUDY IN THREE CENTRES}

doi:10.1136/gut.2011.239301.217

G Lasseter, ${ }^{1}$ S D'Arcy, ${ }^{2}$ A Lawson, ${ }^{3}$ I Shaw, ${ }^{4}$ E Glocker, ${ }^{5}$ C A M Mcnulty, ${ }_{1}^{*}$ 'Primary Care Unit, Health Protection Agency, Gloucester, UK; ${ }^{2}$ Microbiology, Public Health Wales, Bangor, Gwynedd, UK; ${ }^{3}$ Helicobacter Reference Unit, Centre for Infections, Health Protection Agency, London, UK; ${ }^{4}$ Endoscopy Unit, Gloucestershire Hospitals NHS Trust, Gloucester, UK; ${ }^{5}$ Division of Infection and Immunity, School of Life and Medical Sciences, University College London, London, UK

Introduction Helicobacter pylori eradication requires PPI with two antibiotics: amoxicillin, clarithromycin or metronidazole. Treatment failures are increasing and alternative antibiotics include tetracyclines, quinolones and rifabutin. In England in $2008 H$. pylori culture and antibiotic sensitivity was only routinely performed in Gloucester and the Helicobacter Reference Unit (HRU).

Methods Determine the feasibility of H. pylori antimicrobial resistance surveillance, using gastric antral biopsy specimens from routine endoscopies cultured in Gloucester and Bangor and referred to HRU. European standard methods were used for culture and susceptibility by E-tests.

Results Prevalence was low: Bangor 6.6\%, Gloucester 5.5\% and HRU $32.2 \%$. Higher resistance rates in the HRU reflected a greater proportion of referrals 'post treatment'. Resistance rates were: Metronidazole, HRU 87\%, Glos 22\%, Bangor $38 \%$; Clarithromycin, HRU 68\%, Glos 4\%, Bangor 15\%; Levofloxacin, HRU 17\%, Glos 1\%, Bangor 13\%; Rifabutin, HRU 0\%, Glos 2.8\%, Bangor 3.3\%; Amoxicillin, HRU 2.8\%, Glos $0 \%$, Bangor 1.7\%; Tetracycline HRU $<1 \%$, Glos $1.4 \%$, Bangor $0 \%$. Patient history was collected retrospectively for all samples and despite paying endoscopy staff, data collection was poor. Consequently a dedicated staff member in Gloucester PCU completed all data collection.

Conclusion Surveillance is essential as a primary Clarithromycin resistance varies greatly between centres. There is higher resistance post treatment. In future a dedicated clerical officer will be required to collect all data. As prevalence is low, large numbers of biopsy specimens are needed to attain sufficient numbers of culture isolates for antibiotic susceptibility surveillance areas of higher prevalence.

Competing interests None.

Keywords antibiotic resistance, helicobacter pylori, Surveillance. 